\title{
Detecting and Tracking Motion of Myxococcus xanthus Bacteria in Swarms ${ }^{\star}$
}

\author{
Xiaomin Liu ${ }^{1}$, Cameron W. Harvey ${ }^{2}$, Haitao Wang ${ }^{1}$, \\ Mark S. Alber ${ }^{2}$, and Danny Z. Chen ${ }^{1}$ \\ 1 Department of Computer Science \& Engineering, University of Notre Dame, USA \\ 2 Department of Applied and Computational Mathematics and Statistics, \\ University of Notre Dame, USA
}

\begin{abstract}
Automatically detecting and tracking the motion of Myxococcus xanthus bacteria provide essential information for studying bacterial cell motility mechanisms and collective behaviors. However, this problem is difficult due to the low contrast of microscopy images, cell clustering and colliding behaviors, etc. To overcome these difficulties, our approach starts with a level set based pre-segmentation of cell clusters, followed by an enhancement of the rod-like cell features and detection of individual bacterium within each cluster. A novel method based on "spikes" of the outer medial axis is applied to divide touching (colliding) cells. The tracking of cell motion is accomplished by a non-crossing bipartite graph matching scheme that matches not only individual cells but also the neighboring structures around each cell. Our approach was evaluated on image sequences of moving $M$. xanthus bacteria close to the edge of their swarms, achieving high accuracy on the test data sets.
\end{abstract}

\section{Introduction}

Myxococcus xanthus is a rod-shaped, Gram-negative soil bacterium that has become a model organism for the study of multicellular development because of the coordinated collective motion that the bacterial cells exhibit when moving on surfaces [1]. Understanding the biomechanical interactions of bacterial cells during movement, such as collisions and cells moving within clusters, will shed light on the collective motion of cells and ultimately on both the processes of swarming and multicellular-structure formation. An important step towards achieving this goal is to track the cell movement and interactions. However, accurate quantification of cell motion faces a number of challenges, including low image contrast, intra- or inter-frame intensity variations, and the frequent clustering and colliding behaviors of multiple cells.

The current cell tracking methods mainly fall into two basic categories: segmentation-based methods and model-based methods. Model-based algorithms such as the geodesic active contour method [2] have gained popularity in recent

\footnotetext{
* This work was supported in part by NSF under grant CCF-0916606 and by NIH
} under grants R01GM095959 and R01GM100470. 
years due to their flexibility in capturing topological changes such as mitosis. However, such methods cannot avoid merging multiple touching cells, which occur quite frequently in our images. Segmentation-based methods consist of two steps: segmenting individual cells in each image frame and mapping segmented cells between consecutive frames. Once segmented cells are obtained in different frames, the remaining task in fact becomes a certain matching problem that could be solved globally by methods such as optimal bipartite graph matching [3], Softassign Procrustes algorithm [4, etc. But, these matching methods utilize only information of independent cells (e.g., their appearances) as matching criteria, and do not consider relations among different cells, such as the spatial distribution of cells in a neighborhood. Indeed, the relative positions of moving cells and their local structures keep changing from frame to frame, which may not be captured directly by simple graph matching methods (e.g., [5]).

We observed that in image sequences of moving $M$. xanthus cells, the changes of relative cell positions are quite moderate, and thus the spatial structures of cells between consecutive image frames remain relatively similar. Based on this observation, we propose a new tracking algorithm that considers the neighboring cells of every target cell in consecutive frames, and captures their similarity despite of certain changes caused by the cell movement.

In this paper, we present a new method that combines cell shape segmentation and optimal graph matching to overcome the difficulties of tracking $M$. xanthus bacteria. First, cell clusters are identified using a level set method. Then individual cells within clusters are segmented by enhancing the cells' rod-like shape features with the eigenvalues of Hessian matrices. Next, false merging of neighboring cells is separated by an approach based on the structures of the outer medial axis of the cells. Finally, a non-crossing maximum bipartite graph matching scheme is applied to compare cell neighboring structures in consecutive frames, producing the frame-to-frame correspondence in image sequences. Based on the tracking results, we can quantitatively study cell motility mechanisms such as the reversal behavior of the bacteria and how they avoid blocking in cell motions.

\section{Method}

\subsection{Segmentation of Cell Clusters}

The regions of cell clusters in each image frame are first identified by a gradient based level set approach [6] that maintains the regularity during the level set evolution (see Fig. 11). To speed up the process, we assign the initial level set function as a binary image generated as follows. (1) Obtain the shadow area surrounding each bacterium by the method introduced in Sec. 2.2. (2) apply the morphological dilation method to the binary image obtained in Step (1) to cover the entire regions of cells. As shown in Sec. 2.2, the segmentation of cell clusters helps eliminate false positive cell segmentation errors near the cell clusters. 

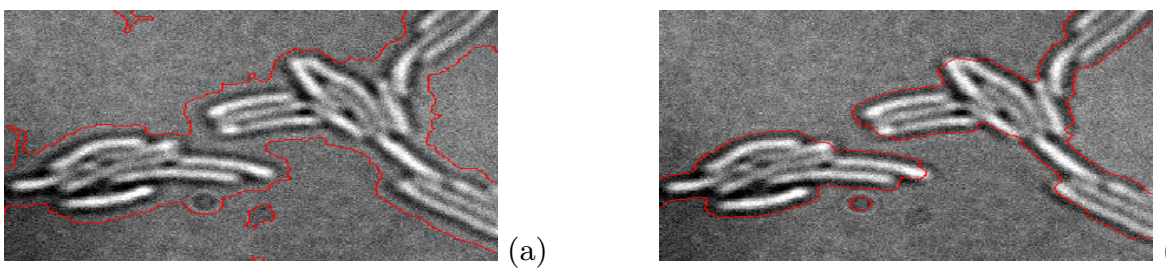

(b)

Fig. 1. An example for the level set segmentation of the cell clusters: (a) The initialization of the level set function (in red); (b) the final segmentation

\subsection{Segmentation of Individual Cells}

The appearance of an $M$. xanthus bacterium in our images is like a bright rodshaped object surrounded by dark shadow area. We propose a method based on the eigenvalues of the Hessian matrix to detect such distinct shape features of each individual cell, which are insensitive to inter- and intra-frame intensity changes. For a 2D image $I$ of bacterial cells, let $I(x, y)$ denote the intensity value at a pixel of coordinates $(x, y)$. We first obtain a smoothed image $L(x, y ; \sigma)$ by convolving $I(x, y)$ with a Gaussian kernel $G(x, y ; \sigma)$, where $\sigma$ is a scale parameter corresponding to the size of the target object [7]. Then the Hessian matrix of a pixel at $(x, y)$ is composed of the second order derivatives of $L: H(x, y)=$ $\left[\begin{array}{ll}L_{x x} & L_{x y} \\ L_{x y} & L_{y y}\end{array}\right]$. We can learn the second order local intensity changes by computing the eigenvalues $\lambda_{1}$ and $\lambda_{2}$ of $H(x, y): \lambda_{1,2}=\frac{L_{x x}+L_{y y} \pm \sqrt{\left(L_{x x}-L_{y y}\right)^{2}+4 L_{x y}^{2}}}{2}$.

If one of the two eigenvalues is close to zero and the other exhibits a high negative or positive value, then it corresponds to a ridge-like local structure. Let $\lambda_{S}$ and $\lambda_{L}$ denote the two eigenvalues such that $\left|\lambda_{S}\right|<\left|\lambda_{L}\right|$, and let $\lambda=\lambda_{S}-\lambda_{L}$. In our approach, we enhance the image for each pixel that has a high positive $\lambda$ value in the rod-like cell regions and a high (absolute) negative $\lambda$ value in the surrounding shadow regions. Those regions are then segmented by thresholding. As shown in Fig. 2(b), a narrow band around each cell cluster also presents high positive $\lambda$ values in the enhanced image, which can be eliminated by the accurate segmentation of the cell cluster boundaries (see Fig. 2 (d)). The scale space based image segmentation algorithm may cause over-partitioning due to intensity changes inside the cells. These cases, which occurred rather infrequently in our images, are mostly near the ends of the cells.

\subsection{Separation of Touching Cells}

The moving bacterial cells frequently touch each other while exhibiting no significant intensity changes around the cell boundaries at the touching areas. The watershed method is commonly used for segmenting touching cells [8]. However, for objects with elongated shapes such as $M$. xanthus bacteria, the watershed method tends to under-segment cell regions and produce too many small fragments. To capture the concavity of the boundary locations where two cells touch, 

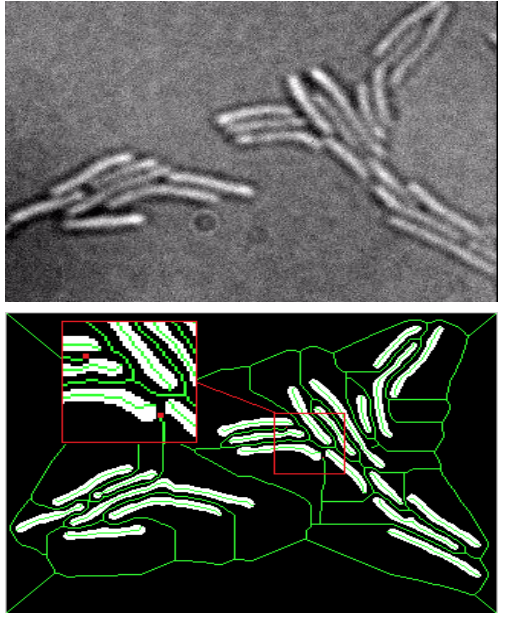

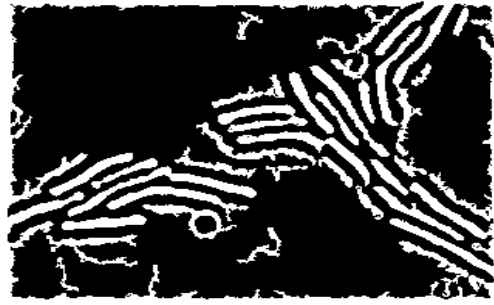

(b)

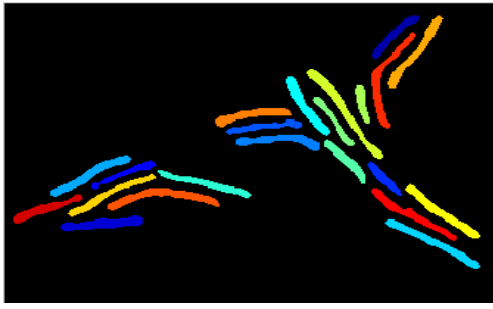

Fig. 2. Illustrating the cell segmentation approach: (a) An original image; (b) cell regions segmented by using the eigenvalues of the Hessian matrix (in Sec. 2.2); (c) cell regions after removing the false positives outside the clusters and separating touching cells based on the outer medial axis (the outer medial axis is in green and the endpoints of the outer medial axis spikes in a boxed area are in red); (d) the final segmentation

a method based on the "spikes" of the outer medial axis of the cell regions is applied to detect the locations of the touching areas [9]. As shown in Fig. 2(c), the outer medial axis of well separated bacteria is similar to their Voronoi diagram. The "spikes" are the segments of the outer medial axis with one disjoint endpoint, which are associated with the boundary concavity between touching cells and actually point at the locations where cell touching occurs.

Fig. 3 illustrates several typical touching cases involving two or more cells (more complex cases can be viewed as combinations of these basic cases). In our settings, almost all touching locations are at the ends of the rod-shaped cells: either two cells merge at two end positions (Fig. 3(a), (c), (d)) or one cell's end touches another cell's body (Fig. 3(b)). After computing the outer medial axis by an iterative thinning method [10, we prune away the false "spikes" that are caused by noisy cell boundaries instead of by true cell touching [9]. Then the endpoints of the remaining spikes are identified and extended along the centerline directions of the spikes to divide the touching cells. An extension line stops if it crosses a neighboring extension line (Fig. 3(b), (c)). If it does not cross any neighboring extension line, then it divides the cell region by itself (Fig. 33(a)).

\subsection{Tracking Cells Based on Non-crossing Maximum Matching of Neighborhood Structures}

Once individual cells are segmented, we map cells in consecutive frames and establish their correspondence throughout an image sequence. For each cell $C$ 

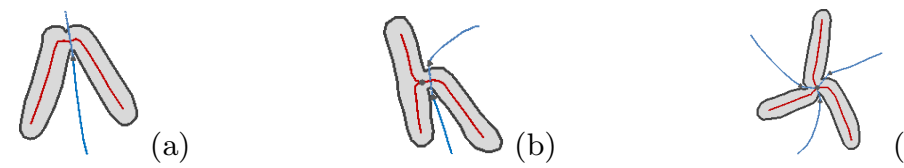

(c)

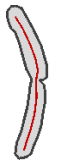

Fig. 3. Examples of separating touching cells based on the outer medial axis: (a) Endto-end touching of two cells; (b) end-to-body touching of two cells; (c) end-to-end touching of multiple cells; (d) a difficult case of end-to-end touching with no spikes presented on the outer medial axis. The outer medial axis is marked in blue and the arrow markers correspond to the endpoints of spikes of the outer medial axis.

in a frame $I_{t}$, we find its corresponding cell $C^{\prime}$ in frame $I_{t+1}$ by comparing the neighboring cells of $C$ in frame $I_{t}$ with those of $C^{\prime}$ in frame $I_{t+1}$, which we model as a bipartite matching problem based on their order around $C$ (and $C^{\prime}$ ). The neighbors of a cell $C$ in a frame are those bacteria whose Voronoi regions share a common edge with the Voronoi region of $C$. Note that since moving cells may enter or leave the neighborhood (i.e., the set of neighbors) of a cell $C$ in consecutive frames, $C$ 's neighborhood in $I_{t}$ may be somewhat different from $C$ 's neighborhood in $I_{t+1}$. For a cell $C_{i}$ in $I_{t}$ and a candidate cell $C_{j}$ of $C_{i}$ in $I_{t+1}$, let $N_{i}$ and $N_{j}$ denote their neighborhoods in $I_{t}$ and $I_{t+1}$ in (say) clockwise cyclic order around $C_{i}$ and $C_{j}$, respectively. We build a bipartite graph $G=(U, V, E)$ with weighted edges, where $U=\left\{u_{1}, u_{2}, \ldots, u_{n_{i}}\right\}$ is for $N_{i}$ and $V=\left\{v_{1}, v_{2}, \ldots, v_{n_{j}}\right\}$ is for $N_{j}$. To capture the similarity between $N_{i}$ and $N_{j}$ that may contain some common cells, we apply a non-crossing bipartite matching algorithm [1] that aims to preserve the order of the neighboring cells while finding a matching with the maximum total edge weight. A non-crossing matching in the graph $G=\{U, V, E\}$ is a subset of edges $M \subseteq E$ such that for any two edges $\left(u_{a}, v_{b}\right)$ and $\left(u_{c}, v_{d}\right)$ in $M$, either $(a<c$ and $b<d)$ or $(c<a$ and $d<b)$ holds. In our problem, it is crucial to obtain a non-crossing maximum matching (instead of just a maximum matching) because an edge crossing corresponds to a wrong order of the matched neighbors which may not reflect the correct neighboring structures. The algorithm in [11] takes $O(m \log n)$ time, where $n=|U|+|V|$ and $m=|E|$.

Our cell mapping algorithm for an image sequence takes the following steps:

1. For the cells in every binary image frame, compute their outer medial axis [10] and associate each cell with its corresponding Voronoi region.

2. In every frame $I_{t}$, for each cell $C_{i}$, identify its neighborhood $N_{i}=\left\{C_{i}^{1}, C_{i}^{2}, \ldots\right.$, $\left.C_{i}^{n_{i}}\right\}$ in clockwise cyclic order around $C_{i}$ (the Voronoi region of every $C_{i}^{k}$ shares a common edge with that of $C_{i}$ ). For a candidate cell $C_{j}$ of $C_{i}$ in frame $I_{t+1}$, let its neighborhood $N_{j}=\left\{C_{j}^{1}, C_{j}^{2}, \ldots, C_{j}^{n_{j}}\right\}$ (see Fig. 4).

3. For each pair $\left(C_{i}^{k}, C_{j}^{l}\right)$ of $C_{i}$ and $C_{j}$, build a bipartite graph $G_{k l}=\{U, V, E\}$, with $U=\left\{C_{i}^{k}, C_{i}^{k+1}, \ldots, C_{i}^{n_{i}}, C_{i}^{1}, \ldots, C_{i}^{k-1}\right\}$ (when $k=1, U=\left\{C_{i}^{1}, C_{i}^{2}, \ldots\right.$, $\left.C_{i}^{n_{i}}\right\}$ ), and $V=\left\{C_{j}^{l}, C_{j}^{l+1}, \ldots, C_{j}^{n_{j}}, C_{j}^{1}, \ldots, C_{j}^{l-1}\right\}$ (when $l=1, U=\left\{C_{j}^{1}, C_{j}^{2}\right.$, $\left.\left.\ldots, C_{j}^{n_{j}}\right\}\right)$. An edge in $E$ connects two vertices $u \in U$ and $v \in V$ if the two corresponding cells satisfy some constraints on their relative distance, their 

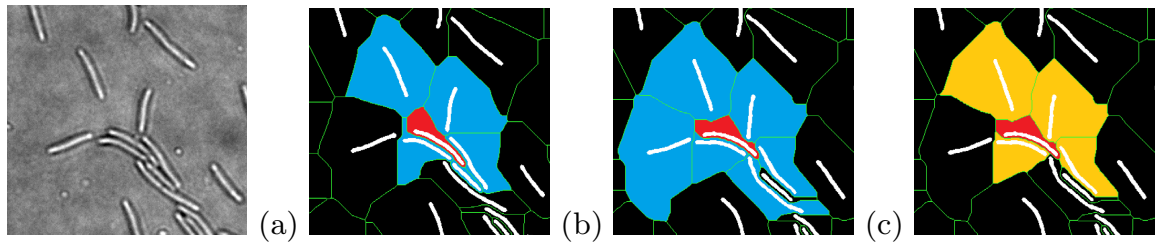

(d)

Fig. 4. Illustrating the non-crossing maximum matching between two sets of neighboring cells: (a) An original image; (b)(c) segmented cell regions and their outer medial axes (in green) in frames $I_{t}$ and $I_{t+1}$, with the center cell in the red region and the neighboring cells in the blue regions; (d) the cells in the non-crossing maximum matching of the two neighboring sets (in the yellow regions)

length difference, and their orientation difference with respect to $C_{i}$ and $C_{j}$; its edge weight $w(u, v)$ is defined by the similarity between the two cells of $u$ and $v, w(u, v)=(c-M H D(u, v)) / c$, where $\operatorname{MHD}(u, v)$ is the modified Hausdorff distance between the two cells of $u$ and $v$, and $c$ is a constant that is two times the maximum cell width. Then we compute the non-crossing maximum weight matching $M_{k l}^{*}$ in $G_{k l}$ using the algorithm in [11. Find the matching $M^{*}$ with the largest weight among all such graphs $G_{k l}$ for $C_{i}$ and $C_{j}$. Add the similarity value $w\left(C_{i}, C_{j}\right)$ for the cells $C_{i}$ and $C_{j}$ to the weight of $M^{*}$, which is the "best" match weight $W\left(C_{i}, C_{j}\right)$ for $C_{i}$ and $C_{j}$. If $W\left(C_{i}, C_{j}\right)$ is smaller than a threshold, then ignore the pair $\left(C_{i}, C_{j}\right)$.

4. Repeat Step 3 for every possible candidate cell $C_{j}$ of $C_{i}$ in frame $I_{t+1}$.

5. For every two frames $I_{t}$ and $I_{t+1}$, sort the remaining "best" match pairs $\left(C_{i}, C_{j}\right)$ based on their "best" match weights $W\left(C_{i}, C_{j}\right)$. Scan the sorted list of "best" match pairs starting from the largest one. Assign correspondence between the paired cells $\left(C_{i}, C_{j}\right)$ in frames $I_{t}$ and $I_{t+1}$. Once a cell $C_{i}\left(C_{j}\right)$ for $I_{t}\left(I_{t+1}\right)$ is assigned a matched cell, all other pairs containing $C_{i}\left(C_{j}\right)$ in $I_{t}$ and $I_{t+1}$ are removed from the list. After the scan, all unmatched cells are viewed as "single" cells (e.g., new cells entering the frames).

\section{Experimental Results}

Swarms of M. xanthus are made on agar plates as detailed in [12. Time-lapse microscopy is performed either on the swarm plates or on specially designed imaging chambers that are similar to the submerged agar chambers (SAC) described in [13. The image size is 512 by 512 . The pixel size is $0.14^{*} 0.07 \mu \mathrm{m}$. The cells move approximately $10-25$ pixels in two consecutive frames. The time interval between two consecutive image frames is 15 seconds.

The experiments were performed on 4 image sequences containing 384 cells in total. Cells were visually tracked in the sequences and compared with the output of our algorithm. The tracking result of one moving cell is considered as correct if the cell is segmented correctly in each frame and the correspondence throughout the entire image sequence is correctly maintained. Incomplete trajectories and 

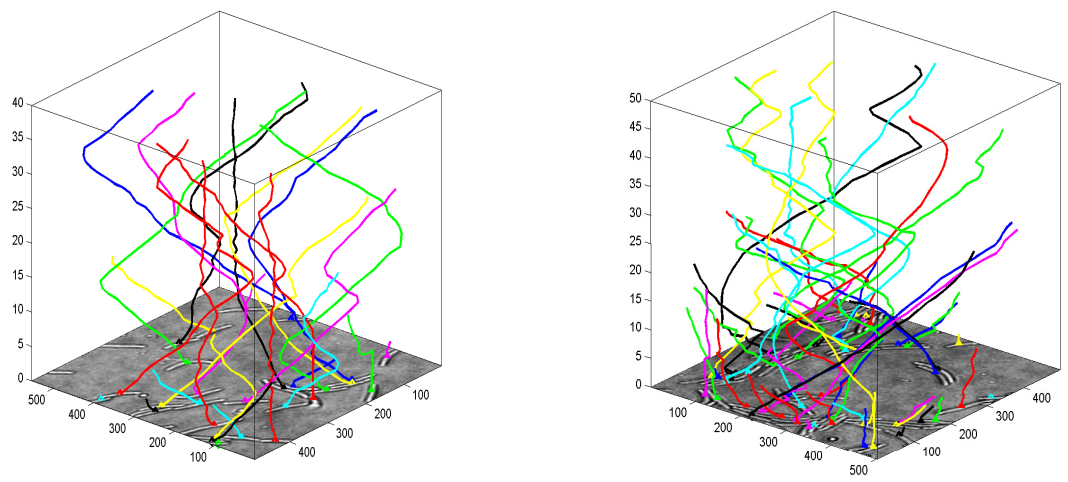

(a)

Fig. 5. Two examples of tracked trajectories of cell centroids in frame stacks, with frame 1 positioned at the $z=0$ plane. Different cells are distinguished by colors.

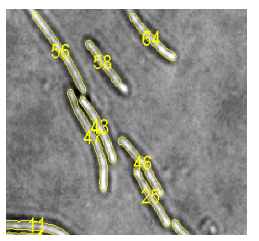

(a)

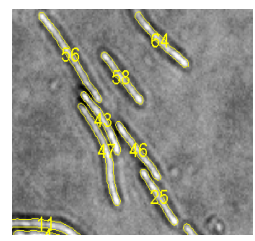

(b)

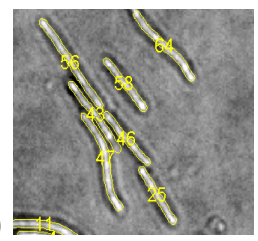

(c)

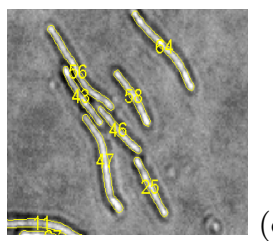

(d)

Fig. 6. Consecutive frames of tracking results

Table 1. The tracking accuracy of cell trajectories

\begin{tabular}{c|c|c|c}
\hline \hline & Independent tracking & With neighborhood & Number of cells \\
\hline Seq1 & $76.9 \%$ & $89.2 \%$ & 65 \\
Seq2 & $71.7 \%$ & $87.1 \%$ & 124 \\
Seq3 & $77.7 \%$ & $92.2 \%$ & 103 \\
Seq4 & $78.3 \%$ & $89.1 \%$ & 92 \\
\hline \hline
\end{tabular}

wrong trajectories are all considered as errors. Fig. 5] shows two examples of tracked cell traces in 3D spatio-temporal space. Fig. 6] is another example of the frame-by-frame correspondence in a magnified region. The tracking errors are mostly due to incorrect segmentation in regions that are blurred when the focal plane of the microscope is being adjusted. Table 1 lists the accuracy of cell trajectories measured in the 4 sequences. It shows our method achieved higher accuracy comparing to the method without utilizing the neighboring structures, which has met the requirement of the current biological application. 


\section{Conclusions}

We present new algorithms for segmenting and tracking $M$. xanthus bacteria that are moving and closely interacting. One of our major contributions is a non-crossing maximum matching method to track moving cells based on their neighborhood structures. The experiments showed high accuracy of tracking cell trajectories by our algorithm. Our approach is applicable to other tracking problems with the assumption that the cell movement is moderate between consecutive image frames, so that the neighboring structure around each cell does not change too substantially from frame to frame (otherwise, it may be meaningless to incorporate the neighboring cell information into the tracking process).

\section{References}

1. Dworkin, M.: Recent advances in the social and developmental biology of the myxobacteria. Microbiological Reviews 60, 70-102 (1996)

2. Li, K., Miller, E.D., Chen, M., Kanade, T., Weiss, L.E., Campbell, P.G.: Cell population tracking and lineage construction with spatiotemporal context. Medical Image Analysis 12, 546-566 (2008)

3. Munkres, J.: Algorithms for the assignment and transportation problems. Journal of the Society for Industrial and Applied Mathematics 5, 32-38 (1957)

4. Gor, V., Elowitz, M., Bacarian, T., Mjolsness, E.: Tracking cell signals in fluorescent images. In: Proc. of the 2005 IEEE Computer Society Conference on Computer Vision and Pattern Recognition - Workshops, p. 142 (2005)

5. Liu, M., Roy-Chowdhury, A.K., Reddy, G.V.: Robust estimation of stem cell lineages using local graph matching. In: IEEE Computer Society Conference on Computer Vision and Pattern Recognition Workshops, pp. 194-201 (2009)

6. Li, C., Xu, C., Gui, C., Fox, M.D.: Distance regularized level set evolution and its application to image segmentation. IEEE Trans. Image Processing 19, 3243-3254 (2010)

7. Lindeberg, T.: Scale-space theory in computer vision. Kluwer Academic Publishers (1994)

8. Vincent, L., Soille, P.: Watersheds in digital spaces: An efficient algorithm based on immersion simulations. IEEE Transactions on Pattern Analysis and Machine Intelligence 13, 583-598 (1991)

9. Liu, X., Setiadi, A.F., Alber, M.S., Lee, P.P., Chen, D.Z.: Identification and classification of cells in multi-spectral microscopy images of lymph node. In: Proceedings of SPIE Medical Imaging: Image Processing, vol. 7962, p. 79620J (2011)

10. Lam, L., Lee, S.W., Suen, C.Y.: Thinning methodologies - A comprehensive survey. IEEE Trans. on Pattern Analysis and Machine Intelligence 14, 869-885 (1992)

11. Malucelli, F., Ottmann, T., Pretolani, D.: Efficient labelling algorithms for the maximum non crossing matching problem. Discrete Applied Mathematics 47, 1-4 (1993)

12. Harvey, C., Morcos, F., Sweet, C.R., Kaiser, K., Chatterjee, S., Liu, X., Chen, D.Z., Alber, M.: Study of elastic collisions of myxococcus xanthus in swarms. Physical Biology 8, 026016 (2011)

13. Welch, R., Kaiser, D.: Cell behavior in traveling wave patterns of myxobacteria. Proc. Natl. Acad. Sci. USA 98, 14907-14912 (2001) 